HUTP-99/A024, NUB 3200, EFI-99-16

\title{
Non-perturbative K3 Orientifolds with NS-NS B-flux
}

\author{
Zurab Kakushadze* \\ Jefferson Laboratory of Physics, Harvard University, Cambridge, MA 02138 \\ and \\ Department of Physics, Northeastern University, Boston, MA 02115 \\ and \\ Enrico Fermi Institute, University of Chicago, Chicago, IL 60637
}

(May 5, 1999)

\begin{abstract}
We consider non-perturbative six dimensional $\mathcal{N}=1$ space-time supersymmetric orientifolds of Type IIB on K3 with non-trivial NS-NS B-flux. All of these models are non-perturbative in both orientifold and heterotic pictures. Thus, some states in such compactifications arise in "twisted" open string sectors which lack world-sheet description in terms of D-branes. We also discuss their dual F-theory compactifications on certain Voisin-Borcea orbifolds. In particular, the explicit construction of non-perturbative K3 orientifolds with NS-NS $B$-flux gives additional evidence for the conjectured extension of Nikulin's classification in the context of Voisin-Borcea orbifolds.
\end{abstract}

Typeset using REVTEX

*E-mail: zurab@string.harvard.edu 
In the recent years various six (see, e.g., [1 4]) and four (see, e.g., [5 [12]) dimensional orientifold vacua have been constructed. In many cases the world-sheet approach to orientifolds is adequate and gives rise to consistent anomaly free vacua in six and four dimensions. However, there are cases where the perturbative orientifold description misses certain nonperturbative sectors giving rise to massless states [11,13,14]. In certain cases this inadequacy results in obvious inconsistencies such as lack of tadpole and anomaly cancellation. Examples of such cases were discussed in [9 11]. In other cases, however, the issue is more subtle as the non-perturbative states arise in anomaly free combinations, so that they are easier to miss.

Recently such non-perturbative orientifolds have been studied in detail in [13, 14]. In particular, six and four dimensional $\mathcal{N}=1$ supersymmetric non-perturbative orientifolds have been constructed in [13,14] using Type I-heterotic duality and the map between Type IIB orientifolds and F-theory as guiding principles. The discussion in [13,14 was (mainly) confined to orientifolds with trivial NS-NS $B$-flux. The purpose of this note is to extend the results of [13,14 to compactifications with non-trivial NS-NS $B$-field turned on. In particular, here we will focus on six dimensional $\mathcal{N}=1$ supersymmetric non-perturbative orientifolds of Type IIB on K3 with non-trivial NS-NS $B$-flux.

The origin of non-perturbative states in orientifold compactifications can be understood as follows. Thus, in the K3 orbifold examples of [3] the orientifold projection is not $\Omega$, which we will use to denote that in the smooth $\mathrm{K} 3$ case, but rather $\Omega J^{\prime}$, where $J^{\prime}$ maps the $g$ twisted sector to its conjugate $g^{-1}$ twisted sector (assuming $g^{2} \neq 1$ ) [15]. Geometrically this can be viewed as a permutation of two $\mathbf{P}^{1}$ 's associated with each fixed point of the orbifold [11]. (More precisely, these $\mathbf{P}^{1}$ 's correspond to the orbifold blow-ups.) This is different from the orientifold projection in the smooth case where (after blowing up) the orientifold projection does not permute the two $\mathbf{P}^{1}$ s. In the case of the $\Omega J^{\prime}$ projection the "twisted" open string sectors corresponding to the orientifold elements $\Omega J^{\prime} g$ are absent [16,11]. However, if the orientifold projection is $\Omega$, then the "twisted" open string sectors corresponding to the orientifold elements $\Omega g$ are present [11. In fact, these states are non-perturbative from the orientifold viewpoint and are required for gravitational anomaly cancellation in six dimensions. This can be explicitly seen from the construction of nonperturbative K3 orientifolds without the $B$-flux in [13, 14.

The effect of turning on non-trivial NS-NS $B$-flux in the perturbative (that is, "untwisted") open string sectors is as follows. First, the rank of the gauge group is reduced by $2^{b / 2}$ [17], where $b$ is the rank of the matrix $B_{i j}(i, j=1,2,3,4$ label the compact directions corresponding to K3). Note that $b$ is always an even integer, and in the case of K3 compactifications can take values $b=0,2,4$. Second, the multiplicity of the 59 open string sector states (if present) is no longer 1 (as in the case without the $B$-field), but $2^{b / 2}[4$. Note that the $B$-field is quantized in half-integer units (here we are using the normalization where the $B$-field is defined modulo integer shifts) [17,4].

The effect of the NS-NS B-flux in the non-perturbative (that is, "twisted") open string sectors is a bit more non-trivial. The point here is that Type I-heterotic duality was an important guiding principle in determining the twisted open string sector spectrum of nonperturbative $\mathrm{K} 3$ orientifolds (e.g., in the case of the $\mathbf{Z}_{3}$ orbifold limit of K3) without the $B$-flux [13]. In particular, in certain cases the corresponding heterotic dual is perturbative, which enables one to understand the non-perturbative sectors on the orientifold side. 
However, as was pointed out in [13], once we turn on the $B$-field, the above property is no longer there - the heterotic duals in these cases are no longer perturbative. This makes understanding twisted open string sectors in the presence of the $B$-flux more involved.

Nonetheless, it turns out that there is a way around this difficulty. The key simplification here is due to the observation of [7.18] (also see [19,20]) that turning on the $B$-field in an orientifold compactification is equivalent to turning on non-commuting Wilson lines. Let us be more precise here. For the sake of definiteness let us consider a compactification on a two-torus $T^{2}$. As was explained in detail in [18], turning on (quantized) $B$-filed of rank $b=2$ on $T^{2}$ is equivalent to modding out by the following freely acting orbifold. Thus, let us turn on two $\mathbf{Z}_{2}$ valued Wilson lines on $T^{2}$ - one corresponding to the $a$-cycle of $T^{2}$, and the other one corresponding to the $b$-cycle. Each of these Wilson lines can be described as a freely acting $\mathbf{Z}_{2}$ orbifold which amounts to shifting the $T^{2}$ lattice by a half-lattice shift along the corresponding cycle of $T^{2}$. In the closed string sector the effect of non-zero $B$-field then corresponds to having non-trivial discrete torsion between the generators, call them $g_{1}$ respectively $g_{2}$, of the two $\mathbf{Z}_{2}$ subgroups. In the open string sector each $\mathbf{Z}_{2}$ has a non-trivial action on the corresponding Chan-Paton charges. This action is described by the $16 \times 16$ Chan-Paton matrices $\gamma_{g_{1}}$ respectively $\gamma_{g_{2}}$. The effect of non-zero $B$-field in the open string sector then corresponds to having non-commuting Chan-Paton matrices: $\gamma_{g_{1}} \gamma_{g_{2}}=-\gamma_{g_{2}} \gamma_{g_{1}}$. That is, even though the (freely acting) orbifold group is $\mathbf{Z}_{2} \otimes \mathbf{Z}_{2}$ (with discrete torsion), the corresponding embedding in the Chan-Paton charges is in terms of the non-Abelian dihedral group $D_{4}$ (see [18] for details).

It is now clear how to deal with turning on the $B$-field in the twisted open string sectors. We start with the cases without the $B$-field, which have been understood in [13, 14], and then mod out by the above freely acting orbifold with the corresponding action on the ChanPaton charges. This procedure is relatively straightforward to carry out, albeit there are some technical subtleties (such as the fact that various relative signs in the corresponding projections require some care) one encounters in the process. One also must take into account not only the original twisted sectors modded out by the above action, but also the additional sectors corresponding to fixed points of the K3 orbifold group element (which is a pure twist) accompanied by the $\mathbf{Z}_{2} \otimes \mathbf{Z}_{2}$ shifts. For the sake of brevity here we will skip (most of) the details of the derivation of the corresponding massless spectra, and simply summarize the results in various tables. We will, however, make various clarifying remarks as we discuss the corresponding models.

Thus, consider Type IIB on $\mathcal{M}_{2}=T^{4} / \mathbf{Z}_{N}$, where the generator $g$ of $\mathbf{Z}_{N}, N=2,3,4,6$, acts on the complex coordinates $z_{1}, z_{2}$ on $T^{4}$ as follows:

$$
g z_{1}=\omega z_{1}, \quad g z_{2}=\omega^{-1} z_{2},
$$

where $\omega \equiv \exp (2 \pi i / N)$. This theory has $\mathcal{N}=2$ supersymmetry in six dimensions.

Next, we consider the $\Omega$ orientifold of this theory. Note that, as we have already mentioned in the previous section, the $\Omega$ projection acts as in the smooth K3 case. This, in particular, implies that we must first blow up the orbifold singularities before orientifolding.

\footnotetext{
${ }^{1}$ Here we work with $16 \times 16$ (rather than $32 \times 32$ ) Chan-Paton matrices for we choose not to count the orientifold images of the corresponding D-branes.
} 
After orientifolding the closed string sector contains the $\mathcal{N}=1$ supergravity multiplet in six dimensions plus the usual tensor supermultiplet. We also have some number $n_{H}^{c}$ of closed string sector hypermultiplets plus some number $\widetilde{n}_{T}$ of extra tensor multiplets. Note that $n_{H}^{c}+\widetilde{n}_{T}=20$ (this follows from the fact that before orientifolding in the $\mathcal{N}=1$ language we have 20 hypermultiplets and 20 tensor multiplets associated with K3). The precise values of $n_{H}^{c}$ and $\widetilde{n}_{T}$, however, depend on the order of the orbifold group $N$ as well as the rank $b$ of the $B$-field.

Thus, in the $\mathbf{Z}_{2}$ case the untwisted closed string sector gives rise to 4 hypermultiplets only. The $\mathbf{Z}_{2}$ twisted closed string sector produces 16 hypermultiplets and no tensor multiplets for $b=0,12$ hypermultiplets and 4 tensor multiplets for $b=2$, and 10 hypermultiplets and

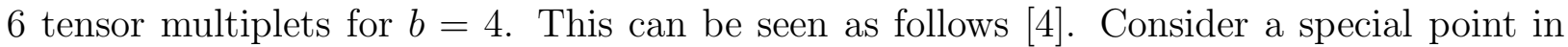
the moduli space where the $T^{4}$ lattice is that of $S O(8)$. In this case the rank of the $B$-field $b=2$. For this special lattice the $\mathbf{Z}_{2}$ twist can be rewritten in terms of lattice shifts (see (414 for details), and in the latter language it is straightforward to see that 12 fixed points are symmetric under the action of $\Omega$, whereas 4 of them are antisymmetric. The above conclusion for the $b=4$ case then follows by considering the four-torus of the factorized form $T^{2} \otimes T^{2}$ and turning on rank $2 B$-field in each $T^{2}$. For each $T^{2}$ the symmetric and antisymmetric fixed points can be deduced from the $b=2$ case discussed above as follows. First consider the case where we have $T^{2} \otimes T^{2}$ with rank $2 B$-field in the first $T^{2}$ and no $B$-field in the second $T^{2}$. Then the second $T^{2}$ gives 4 fixed points which are symmetric under $\Omega$. Since we know that we must have total of 12 symmetric and 4 antisymmetric fixed points for $b=2$, it then follows that the first $T^{2}$ gives 3 symmetric and 1 antisymmetric fixed points. Now it is clear that in the case where both $T^{2}$ 's have rank $2 B$-field turned on, there are total 10 symmetric and 6 antisymmetric fixed points.

Next, let us consider other $\mathbf{Z}_{N}$ cases with $N=3,4,6$. In the untwisted sector we have 2 hypermultiplets and no tensor multiplets. For $N=4,6$ we have the $\mathbf{Z}_{2}$ twisted sector. As was discussed in [1], after the appropriate projections, in the $\mathbf{Z}_{2}$ sector we have: 10 symmetric and no antisymmetric fixed points for $N=4, b=0 ; 8$ symmetric and 2 antisymmetric fixed points for $N=4, b=2 ; 7$ symmetric and 3 antisymmetric fixed points for $N=4, b=4 ; 6$ symmetric and no antisymmetric fixed points for $N=6$ and all three values of $b=0,2,4$. Finally, in the $\mathbf{Z}_{N}$ twisted sectors with $N=3,4,6$ the fixed points are always symmetric under $\Omega$. This can be seen, say, for the $\mathbf{Z}_{3}$ case by going to the special point in the moduli space where $T^{4}$ factorizes as $T^{2} \otimes T^{2}$ with each $T^{2}$ corresponding to the $S U(3)$ lattice. In this case each $T^{2}$ has rank $2 B$-field turned on. For the $S U(3)$ lattice the $\mathbf{Z}_{3}$ twist can be rewritten in terms of the corresponding $\mathbf{Z}_{3}$ valued shift, and then it is not difficult to see that all three fixed points in each $T^{2}$ are symmetric under $\Omega$.

Putting all of the above together, we conclude that for the non-perturbative $\Omega$ orientifolds of Type IIB on K3 we have: $n_{H}^{c}=20, \widetilde{n}_{T}=0$ for $N=2, b=0 ; n_{H}^{c}=16, \widetilde{n}_{T}=4$ for $N=2, b=2 ; n_{H}^{c}=14, \widetilde{n}_{T}=6$ for $N=2, b=4 ; n_{H}^{c}=20, \widetilde{n}_{T}=0$ for $N=3,6, b=0,2,4$; $n_{H}^{c}=20, \widetilde{n}_{T}=0$ for $N=4, b=0 ; n_{H}^{c}=18, \widetilde{n}_{T}=2$ for $N=4, b=2 ; n_{H}^{c}=17, \widetilde{n}_{T}=3$ for $N=4, b=4$. This is reflected in the corresponding tables.

Let us now turn to the open string sector. In the $\mathbf{Z}_{3}$ case the twisted Chan-Paton matrices are completely fixed by the tadpole cancellation conditions for each value of $b$. The corresponding perturbative spectra can be found in [4]. As to the non-perturbative $\mathbf{Z}_{3}$ twisted 99 open string sector, in the $b=0$ case it was given in [13]. To obtain the 
corresponding non-perturbative sector states for $b=2,4$, we mod out by the freely acting orbifold action discussed above. The massless spectra of the $\mathbf{Z}_{3}$ non-perturbative orientifolds with various values of $b$ are summarized in Table $\mathrm{I}$.

In the $\mathbf{Z}_{N}$ cases with $N=2,4,6$ the tadpole cancellation conditions do not uniquely fix the twisted Chan-Paton matrices. More precisely, in the $\mathbf{Z}_{2}$ case with $b=0$ we have a unique (up to equivalent representations) solution for $\gamma_{R}$, where $R$ is the generator of $\mathbf{Z}_{2}$ :

$$
\gamma_{R}=\operatorname{diag}\left(i \mathbf{I}_{8},-i \mathbf{I}_{8}\right)
$$

where $\mathbf{I}_{n}$ denotes the unit $n \times n$ matrix. (Note that the action of the orbifold group on the D9- and D5-branes is similar.) However, as pointed out in [14, for $b \neq 0$ we have two solutions:

$$
\begin{aligned}
& \gamma_{R}=\operatorname{diag}\left(i \mathbf{I}_{2^{3-b / 2}},-i \mathbf{I}_{2^{3-b / 2}}\right), \\
& \gamma_{R}=\operatorname{diag}\left(\mathbf{I}_{2^{3-b / 2}},-\mathbf{I}_{2^{3-b / 2}}\right),
\end{aligned}
$$

where the first solution corresponds to the case without vector structure [21,22], whereas the second solution corresponds to the case with vector structure [14].

In the $\mathbf{Z}_{2}$ case there are no non-perturbative twisted open string sectors, and the corresponding massless spectra for various values of $b$ can be found in [4] for the cases without vector structure, and in [13] for the cases with vector structure. Here we will therefore focus on the $\mathbf{Z}_{4}$ and $\mathbf{Z}_{6}$ cases with and without vector structure. In the cases without vector structure the corresponding twisted massless spectra can be worked out by starting from the $b=0$ case and modding out by the freely acting orbifold action discussed above. The resulting massless spectra for the $\mathbf{Z}_{4}$ orbifold models are given in Table I. In Table II we summarize the massless spectra for the $\mathbf{Z}_{6}$ orbifold models without vector structure. Note that all of these models satisfy the gravitational anomaly cancellation condition [23]

$$
n_{H}-n_{V}=273-29 n_{T}
$$

where $n_{H}, n_{V}$ and $n_{T}$ are the total numbers of hypermultiplets, vector multiplets and tensor multiplets, respectively. (Note that $n_{T}=\widetilde{n}_{T}+1$.)

Next, we turn to the $\mathbf{Z}_{4}$ and $\mathbf{Z}_{6}$ cases with vector structure. Here we must have $b=2$ or 4 . Note that the perturbative open string spectrum of the $\mathbf{Z}_{6}$ model with $b=2$ with vector structure is the same as that of the $\mathbf{Z}_{6}$ model with $b=2$ without vector structure [14]. In fact, it is not difficult to show that the non-perturbative twisted open string spectra are also the same in these two cases. The massless spectrum of the $\mathbf{Z}_{6}$ case with $b=4$ with vector structure is summarized in Table III. Finally, the massless spectra of the $\mathbf{Z}_{4}$ models with $b=2$ and $b=4$ with vector structure are also given in Table III. Note that all of the models in Table III also satisfy the gravitational anomaly cancellation condition (5). As to the Abelian anomalies present in models with $U(1)$ factors, they are expected to be canceled via the generalized Green-Schwarz mechanism [24,21.

In the remainder of this note we would like to discuss F-theory duals of the above non-perturbative orientifolds with NS-NS $B$-flux. In order to understand these F-theory compactifications, we will take an indirect route. Let us further compactify a given nonperturbative K3 orientifold on $T^{2}$. The resulting four dimensional model has $\mathcal{N}=2$ supersymmetry. Let us go to a generic point in the moduli space where the gauge group 
is maximally Higgsed (so that the gauge group is either completely broken or consists of Abelian factors only) 2 . Let $r(V)$ be the number of open string vector multiplets after Higgsing. Then the total number of vector multiplets is given by $r(V)+n_{T}+2$, where $n_{T}$ is the number of tensor multiplets in six dimensions. Let $H^{0}$ be the number of hypermultiplets neutral with respect to the left-over Abelian gauge group (if any). Then, if we assume that the resulting four dimensional model has a Type IIA dual, the Hodge numbers of the corresponding Calabi-Yau three-fold are given by [26]:

$$
\begin{aligned}
& h^{1,1}=r(V)+n_{T}+2, \\
& h^{2,1}=H^{0}-1 .
\end{aligned}
$$

Using various dualities between Type IIA, heterotic, Type I and F-theory, it is not difficult to see that these Calabi-Yau three-folds must be elliptically fibered, and F-theory compactifications on these spaces should be dual to the original six dimensional orientifold models. In the table below we give the Hodge (and Euler) numbers of the Calabi-Yau three-folds for the perturbative (that is, $\Omega J^{\prime}$ ) K3 orientifolds as well as non-perturbative (that is, $\Omega$ ) K3 orientifolds with various values of $b$ (note that in the $\mathbf{Z}_{2}$ case the $\Omega J^{\prime}$ and $\Omega$ orientifolds are equivalent as the action of $J^{\prime}$ is trivial):

\begin{tabular}{|c|c|c|c|c|}
\hline Model & $b$ & $\left(h^{1,1}, h^{2,1}\right)$ & $\chi$ & $(r, a)$ \\
\hline $\mathbf{Z}_{2}, \Omega$ & 0 & $(3,243)$ & -480 & $(2,4)$ \\
\hline & 2 & $(7,127)$ & -240 & $(6,8)$ \\
\hline & 4 & $(9,69)$ & -120 & $(8,10)$ \\
\hline $\mathbf{Z}_{3}, \Omega J^{\prime}$ & 0 & $(20,14)$ & 12 & $(11,9)$ \\
\hline & 2 & $(16,10)$ & 12 & $(11,11)$ \\
\hline & 4 & $(16,10)$ & 12 & $(11,11)$ \\
\hline $\mathbf{Z}_{4}, \Omega J^{\prime}$ & 0 & $(7,127)$ & -240 & $(6,8)$ \\
\hline & 2 & $(9,69)$ & -120 & $(8,10)$ \\
\hline & 4 & $(10,40)$ & -60 & $(9,11)$ \\
\hline $\mathbf{Z}_{6}, \Omega J^{\prime}$ & 0 & $(9,69)$ & -120 & $(6,8)$ \\
\hline & 2 & $(9,69)$ & -120 & $(6,8)$ \\
\hline & 4 & $(9,69)$ & -120 & $(6,8)$ \\
\hline $\mathbf{Z}_{3}, \Omega$ & 0 & $(11,59)$ & -96 & $(2,0)$ \\
\hline & 2 & $(7,55)$ & -96 & $(2,2)$ \\
\hline & 4 & $(7,55)$ & -96 & $(2,2)$ \\
\hline $\mathbf{Z}_{4}, \Omega$ & 0 & $(3,243)$ & -480 & $(2,4)$ \\
\hline & 2 & $(5,185)$ & -360 & $(4,6)$ \\
\hline & 4 & $(6,156)$ & -300 & $(5,7)$ \\
\hline $\mathbf{Z}_{6}, \Omega$ & 0 & $(3,243)$ & -480 & $(2,4)$ \\
\hline & 2 & $(3,243)$ & -480 & $(2,4)$ \\
\hline & 4 & $(3,243)$ & -480 & $(2,4)$ \\
\hline
\end{tabular}

\footnotetext{
${ }^{2}$ More concretely, first we maximally Higgs using the hypermultiplet matter, and then use the adjoint scalars in the $\mathcal{N}=2$ vector multiplets to break any remaining non-Abelian group to its Cartan subalgebra [25].
} 
Note that the above table covers the cases with and without vector structure alike. In the fourth column we have displayed the Euler number $\chi=2\left(h^{1,1}-h^{2,1}\right)$. The meaning of the last column will become clear in a moment.

All of the above Calabi-Yau three-folds correspond to Voisin-Borcea orbifolds [27,28] (for a physicists discussion, see, e.g., [29,30]). Here we will therefore briefly review some facts about Voisin-Borcea orbifolds.

Thus, let $\mathcal{W}_{2}$ be a K3 surface (which is not necessarily an orbifold of $T^{4}$ ) which admits an involution $J$ such that it reverses the sign of the holomorphic two-form $\Omega_{2}$ on $\mathcal{W}_{2}$. Consider the following quotient:

$$
\mathcal{Y}_{3}=\left(T^{2} \otimes \mathcal{W}_{2}\right) / Y
$$

where $Y=\{1, S\} \approx \mathbf{Z}_{2}$, and $S$ acts as $S z_{0}=-z_{0}$ on $T^{2}\left(z_{0}\right.$ being a complex coordinate on $T^{2}$, and as $J$ on $\mathcal{W}_{2}$. This quotient, known as a Voisin-Borcea orbifold, is a CalabiYau three-fold with $S U(3)$ holonomy which is elliptically fibered over the base $\mathcal{B}_{2}=\mathcal{W}_{2} / B$, where $B=\{1, J\} \approx \mathbf{Z}_{2}$.

Nikulin gave a classification [31] of possible involutions of K3 surfaces in terms of three invariants $(r, a, \delta)$. The result of this classification is plotted in Fig.1 (which has been borrowed from [11]) according to the values of $r$ and $a$. The open and closed circles correspond to the cases with $\delta=0$ and $\delta=1$, respectively. (The cases denoted by " $\otimes$ " are outside of Nikulin's classification, and we will discuss them shortly.) In the case $(r, a, \delta)=(10,10,0)$ the base $\mathcal{B}_{2}$ is an Enriques surface, and the corresponding $\mathcal{Y}_{3}$ has Hodge numbers $\left(h^{1,1}, h^{2,1}\right)=(11,11)$. In all the other cases the Hodge numbers are given by:

$$
\begin{aligned}
& h^{1,1}=5+3 r-2 a, \\
& h^{2,1}=65-3 r-2 a .
\end{aligned}
$$

For $(r, a, \delta)=(10,10,0)$ the $\mathbf{Z}_{2}$ twist $S$ is freely acting (that is, it has no fixed points). For $(r, a, \delta)=(10,8,0)$ the fixed point set of $S$ consists of two curves of genus 1 . The base $\mathcal{B}_{2}$ in this case is $\mathbf{P}^{2}$ blown up at 9 points. In all the other cases the fixed point set of $S$ consists of one curve of genus $g$ plus $k$ rational curves, where

$$
\begin{aligned}
g & =\frac{1}{2}(22-r-a), \\
k & =\frac{1}{2}(r-a) .
\end{aligned}
$$

Note that except for the cases with $a=22-r, r=11, \ldots, 20$, the mirror pair of $\mathcal{Y}_{3}$ is given by the Voisin-Borcea orbifold $\widetilde{\mathcal{Y}}_{3}$ with $\widetilde{r}=20-r, \widetilde{a}=a$. Under the mirror transform we have: $\widetilde{g}=f, \widetilde{f}=g$, where $f \equiv k+1$.

In the cases $a=22-r, r=11, \ldots, 20$, the mirror would have to have $\widetilde{r}=20-r$ and $\widetilde{a}=a=\widetilde{r}+2$, where $\widetilde{r}=0, \ldots, 9$. We have depicted these cases in Fig.2 using the " $\otimes$ " symbol. In particular, we have plotted cases with $a=r+2, r=0, \ldots, 10$. The Hodge numbers for these cases are still given by (9) and (10) (which follows from their definition as mirror pairs of the cases with $a=22-r, r=11, \ldots, 20$ ). (This is true for $a=r+2, r=0, \ldots, 9$. Extrapolation to $r=10$ is motivated by the fact that in this case we get $\left(h^{1,1}, h^{2,1}\right)=(11,11)$ which is the same as for $(r, a, \delta)=(10,10,0)$.) In [11] it 
was argued that these Voisin-Borcea orbifolds also exist, albeit they are singular. In fact, some of them can be constructed explicitly (see [11] for details). For instance, the case with $r=2, a=4$ has Hodge numbers $\left(h^{1,1}, h^{2,1}\right)=(3,51)$, which correspond to the singular Calabi-Yau realized as the $\mathbf{Z}_{2} \otimes \mathbf{Z}_{2}$ orbifold with discrete torsion 33,33 .

As we have already mentioned, here we would like to briefly review F-theory compactifications on Voisin-Borcea orbifolds (which correspond to $\mathcal{N}=1$ supersymmetric vacua in six dimensions). Thus, consider F-theory on $\mathcal{Y}_{3}$ with $(r, a, \delta) \neq(10,10,0)$ or $(10,8,0)$. This gives rise to the following massless spectrum in six dimensions. The number of tensor multiplets is $T=r-1$. The number of neutral hypermultiplets is $H=22-r$. The gauge group is $S O(8) \otimes S O(8)^{k}$. There are $g$ adjoint hypermultiplets of the first $S O(8)$. There are no hypermultiplets charged under the other $k S O(8)$ 's. Under mirror symmetry $g$ and $f=k+1$ are interchanged. Thus, the vector multiplets in the adjoint of $S O(8)^{k}$ are traded for $g-1$ hypermultiplets in the adjoint of the first $S O(8)$. That is, gauge symmetry turns into global symmetry and vice-versa. This can be pushed further to understand F-theory compactifications on Calabi-Yau three-folds with $a=r+2, r=1, \ldots, 10$, which give the following spectra. The number of tensor multiplets is $T=r-1$. There are $H=22-r$ neutral hypermultiplets. In addition there are $g=10-r$ hypermultiplets transforming as adjoints under a global $S O(8)$ symmetry. There are no gauge bosons, however. It is not difficult to verify that this massless spectrum is free of gravitational anomalies in six dimensions. In fact, in [11] it was conjectured that all of these singular Calabi-Yau three-folds exist, and F-theory compactifications on these singular spaces are equivalent to F-theory compactifications on smooth Calabi-Yau three-folds according to the following relation [11]:

$$
\begin{aligned}
& \text { F-theory on } \mathcal{Y}_{3} \text { with }\left(h^{1,1}, h^{2,1}\right)=(r+1,61-5 r) \text { is equivalent to } \\
& \text { F-theory on } \widehat{\mathcal{Y}}_{3} \text { with }\left(\hat{h}^{1,1}, \hat{h}^{2,1}\right)=(r+1,301-29 r)(r=1, \ldots, 9) \text {. }
\end{aligned}
$$

Thus, for instance, for $r=2$ we get $\left(\hat{h}^{1,1}, \hat{h}^{2,1}\right)=(3,243)$, which is the elliptic Calabi-Yau given by the $T^{2}$ fibration over the base $\mathbf{P}^{1} \otimes \mathbf{P}^{1}$.

In the above discussion of perturbative K3 orientifolds we have encountered Calabi-Yau three-folds with the Hodge numbers $\left(\hat{h}^{1,1}, \hat{h}^{2,1}\right)=(3,243),(7,127),(9,69),(10,40)$, and also $\left(h^{1,1}, h^{2,1}\right)=(20,14),(16,10)$ (see the above table). The last two cases are within Nikulin's classification, and correspond to $(r, a)=(11,9)$ and $(11,11)$, respectively. The first four cases, however, are outside of Nikulin's classification, and correspond to the above CalabiYau three-folds $\widehat{\mathcal{Y}}_{3}$ (whose Hodge numbers are given by $\left(\hat{h}^{1,1}, \hat{h}^{2,1}\right)=(r+1,301-r)$ ) with $r=2,6,8,9$, respectively. The cases $r=6,8$ were originally discussed in [35]. In fact, in 36] the Calabi-Yau three-fold with $\left(\hat{h}^{1,1}, \hat{h}^{2,1}\right)=(7,127)$ (corresponding to $r=6$ ) was explicitly constructed. Here we also have an additional case with $r=9$. The fact that perturbative K3 orientifolds with NS-NS $B$-field are consistent string backgrounds gives evidence for the conjectured extension of Nikulin's classification.

In fact, in our discussion of non-perturbative $\mathrm{K} 3$ orientifolds we have encountered four new cases: $\left(\hat{h}^{1,1}, \hat{h}^{2,1}\right)=(5,185),(6,156)$, and $\left(h^{1,1}, h^{2,1}\right)=(11,59),(7,55)$. The first two cases correspond to the $\widehat{\mathcal{Y}}_{3}$ three-folds with $r=4,5$, respectively. Our construction of nonperturbative K3 orientifolds with the NS-NS $B$-flux therefore gives additional evidence for

\footnotetext{
${ }^{3}$ For a discussion of F-theory compactifications on mirror manifolds, see, e.g., [34].
} 
the above conjecture. As to the other two cases, they correspond to Voisin-Borcea orbifolds (within Nikulin's classification) with $(r, a)=(2,0)$ (in this case the base is the Hirzebruch surface $\mathbf{F}_{4}$ ) respectively $(r, a)=(2,2)$ (in which case the base is either $\mathbf{F}_{0}$ or $\mathbf{F}_{1}$ ).

Before we end this note, we would like to point out that, having understood nonperturbative K3 orientifolds with NS-NS $B$-flux, it is now straightforward to construct non-perturbative orientifolds on $T^{6} / \Gamma$ orbifolds with $S U(3)$ holonomy in the presence of NS-NS $B$-flux along the lines of [13, 114].

This work was supported in part by the grant NSF PHY-96-02074, and the DOE 1994 OJI award. I would also like to thank Albert and Ribena Yu for financial support. 


\section{TABLES}

\begin{tabular}{|c|c|c|c|c|c|}
\hline Model & $b$ & Gauge Group & $\begin{array}{c}\text { Charged } \\
\text { Hypermultiplets }\end{array}$ & $\begin{array}{c}\text { Neutral } \\
\text { Hypermultiplets }\end{array}$ & $\begin{array}{c}\text { Extra Tensor } \\
\text { Multiplets }\end{array}$ \\
\hline \multirow[t]{3}{*}{$\mathbf{Z}_{3}$} & 0 & {$[U(8) \otimes S O(16)]_{99}$} & $\begin{array}{l}\mathbf{2 8}, \mathbf{1})_{U} \\
(\mathbf{8}, \mathbf{1 6})_{U} \\
9 \times(\mathbf{2 8}, \mathbf{1})_{T}\end{array}$ & 20 & 0 \\
\hline & 2 & $\overline{U(8)_{99}}$ & $\begin{array}{l}\mathbf{3 6}_{U} \\
9 \times \mathbf{2 8}_{T}\end{array}$ & 20 & 0 \\
\hline & 4 & $S O(8)_{99}$ & $9 \times \mathbf{2 8}_{T}$ & 20 & 0 \\
\hline \multirow[t]{2}{*}{$\mathbf{Z}_{4}$} & 0 & $\begin{array}{c}{[U(8) \otimes U(8)]_{99} \otimes} \\
{[U(8) \otimes U(8)]_{55}}\end{array}$ & $\begin{array}{l}(\mathbf{2 8}, \mathbf{1} ; \mathbf{1}, \mathbf{1})_{U} \\
(\mathbf{1}, \mathbf{2 8} ; \mathbf{1}, \mathbf{1})_{U} \\
(\mathbf{8}, \mathbf{8} ; \mathbf{1}, \mathbf{1})_{U} \\
(\mathbf{2 8}, \mathbf{1} ; \mathbf{1}, \mathbf{1})_{T} \\
(\mathbf{1}, \mathbf{2 8} ; \mathbf{1}, \mathbf{1})_{T} \\
\text { same as above with } 99 \leftrightarrow 55 \\
(\mathbf{8}, \mathbf{1} ; \mathbf{8}, \mathbf{1})_{U} \\
(\mathbf{1}, \mathbf{8} ; \mathbf{1}, \mathbf{8})_{U}\end{array}$ & 20 & 0 \\
\hline & 2 & $\begin{array}{c}{[U(4) \otimes U(4)]_{99} \otimes} \\
{[U(4) \otimes U(4)]_{55}}\end{array}$ & $\begin{array}{l}(\mathbf{6}, \mathbf{1} ; \mathbf{1}, \mathbf{1})_{U} \\
(\mathbf{1}, \mathbf{6} ; \mathbf{1}, \mathbf{1})_{U} \\
(\mathbf{4}, \mathbf{4} ; \mathbf{1}, \mathbf{1})_{U} \\
(\mathbf{1 0}, \mathbf{1} ; \mathbf{1}, \mathbf{1})_{T} \\
3 \times(\mathbf{6}, \mathbf{1} ; \mathbf{1}, \mathbf{1})_{T} \\
(\mathbf{1}, \mathbf{1 0} ; \mathbf{1}, \mathbf{1})_{T} \\
3 \times(\mathbf{1}, \mathbf{6} ; \mathbf{1}, \mathbf{1})_{T} \\
\text { same as above with } 99 \leftrightarrow 55 \\
2 \times(\mathbf{4}, \mathbf{1} ; \mathbf{4}, \mathbf{1})_{U} \\
2 \times(\mathbf{1}, \mathbf{4} ; \mathbf{1}, \mathbf{4})_{U}\end{array}$ & 18 & 2 \\
\hline & 4 & $\begin{array}{c}{[U(2) \otimes U(2)]_{99} \otimes} \\
{[U(2) \otimes U(2)]_{55}}\end{array}$ & $\begin{array}{l}4 \times(\mathbf{1}, \mathbf{1} ; \mathbf{1}, \mathbf{1})_{U} \\
(\mathbf{2}, \mathbf{2} ; \mathbf{1}, \mathbf{1})_{U} \\
6 \times(\mathbf{3}, \mathbf{1} ; \mathbf{1}, \mathbf{1})_{T} \\
10 \times(\mathbf{1}, \mathbf{1} ; \mathbf{1}, \mathbf{1})_{T} \\
6 \times(\mathbf{1}, \mathbf{3} ; \mathbf{1}, \mathbf{1})_{T} \\
10 \times(\mathbf{1}, \mathbf{1} ; \mathbf{1}, \mathbf{1})_{T} \\
\text { same as above with } 99 \leftrightarrow 55 \\
4 \times(\mathbf{2}, \mathbf{1} ; \mathbf{2}, \mathbf{1})_{U} \\
4 \times(\mathbf{1}, \mathbf{2} ; \mathbf{1}, \mathbf{2})_{U}\end{array}$ & 17 & 3 \\
\hline
\end{tabular}

TABLE I. The massless spectra of the non-perturbative Type IIB orientifolds on $T^{4} / \mathbf{Z}_{N}$ for $N=3,4$ with the NS-NS $B$-field of rank $b$. The semi-colon in the column "Charged Hypermultiplets" separates 99 and 55 representations. The subscript " $U$ " indicates that the corresponding ("untwisted") state is perturbative from the orientifold viewpoint. The subscript " $T$ " indicates that the corresponding ("twisted") state is non-perturbative from the orientifold viewpoint. The $U(1)$ charges are not shown, and by "neutral" hypermultiplets we mean that the corresponding states are not charged under the non-Abelian subgroups. 


\begin{tabular}{|c|c|c|c|c|c|}
\hline Model & $b$ & Gauge Group & $\begin{array}{c}\text { Charged } \\
\text { Hypermultiplets }\end{array}$ & \begin{tabular}{c|} 
Neutral \\
Hypermultiplets
\end{tabular} & $\begin{array}{c}\text { Extra Tensor } \\
\text { Multiplets }\end{array}$ \\
\hline \multirow[t]{3}{*}{$\mathbf{Z}_{6}$} & 0 & $\begin{array}{c}{[U(4) \otimes U(4) \otimes U(8)]_{99} \otimes} \\
{[U(4) \otimes U(4) \otimes U(8)]_{55}}\end{array}$ & $\begin{array}{l}(\mathbf{6}, \mathbf{1}, \mathbf{1} ; \mathbf{1}, \mathbf{1}, \mathbf{1})_{U} \\
(\mathbf{1}, \mathbf{6}, \mathbf{1} ; \mathbf{1}, \mathbf{1}, \mathbf{1})_{U} \\
(\mathbf{4}, \mathbf{1}, \mathbf{8} ; \mathbf{1}, \mathbf{1}, \mathbf{1})_{U} \\
(\mathbf{1}, \mathbf{4}, \mathbf{8} ; \mathbf{1}, \mathbf{1}, \mathbf{1})_{U} \\
\text { same as above with } 99 \leftrightarrow 55 \\
(\mathbf{4}, \mathbf{1}, \mathbf{1} ; \mathbf{4}, \mathbf{1}, \mathbf{1})_{U} \\
(\mathbf{1}, \mathbf{4}, \mathbf{1} ; \mathbf{1}, \mathbf{4}, \mathbf{1})_{U} \\
(\mathbf{1}, \mathbf{1}, \mathbf{8} ; \mathbf{1}, \mathbf{1}, \mathbf{8})_{U} \\
5 \times(\mathbf{6}, \mathbf{1}, \mathbf{1} ; \mathbf{1}, \mathbf{1}, \mathbf{1})_{T} \\
5 \times(\mathbf{1}, \mathbf{6}, \mathbf{1} ; \mathbf{1}, \mathbf{1}, \mathbf{1})_{T} \\
4 \times(\mathbf{4}, \mathbf{4}, \mathbf{1} ; \mathbf{1}, \mathbf{1}, \mathbf{1})_{T} \\
(\mathbf{1}, \mathbf{1}, \mathbf{1} ; \mathbf{6}, \mathbf{1}, \mathbf{1})_{T} \\
(\mathbf{1}, \mathbf{1}, \mathbf{1} ; \mathbf{1}, \mathbf{6}, \mathbf{1})_{T} \\
(\mathbf{4}, \mathbf{1}, \mathbf{1} ; \mathbf{4}, \mathbf{1}, \mathbf{1})_{T} \\
(\mathbf{1}, \mathbf{4}, \mathbf{1} ; \mathbf{1}, \mathbf{4}, \mathbf{1})_{T}\end{array}$ & 20 & 0 \\
\hline & 2 & $\begin{array}{l}{[U(4) \otimes U(4)]_{99} \otimes} \\
{[U(4) \otimes U(4)]_{55}}\end{array}$ & $\begin{array}{l}(\mathbf{6}, \mathbf{1} ; \mathbf{1}, \mathbf{1})_{U} \\
(\mathbf{1}, \mathbf{6} ; \mathbf{1}, \mathbf{1})_{U} \\
(\mathbf{4}, \mathbf{4} ; \mathbf{1}, \mathbf{1})_{U} \\
\text { same as above with } 99 \leftrightarrow 55 \\
2 \times(\mathbf{4}, \mathbf{1} ; \mathbf{4}, \mathbf{1})_{U} \\
2 \times(\mathbf{1}, \mathbf{4} ; \mathbf{1}, \mathbf{4})_{U} \\
5 \times(\mathbf{6}, \mathbf{1} ; \mathbf{1}, \mathbf{1})_{T} \\
5 \times(\mathbf{1}, \mathbf{6} ; \mathbf{1}, \mathbf{1})_{T} \\
4 \times(\mathbf{4}, \mathbf{4} ; \mathbf{1}, \mathbf{1})_{T} \\
(\mathbf{1}, \mathbf{1} ; \mathbf{6}, \mathbf{1})_{T} \\
(\mathbf{1}, \mathbf{1} ; \mathbf{1}, \mathbf{6})_{T} \\
(\mathbf{4}, \mathbf{1} ; \mathbf{4}, \mathbf{1})_{T} \\
(\mathbf{1}, \mathbf{4} ; \mathbf{1}, \mathbf{4})_{T}\end{array}$ & 20 & 0 \\
\hline & 4 & $U(4)_{99} \otimes U(4)_{55}$ & $\begin{array}{l}2 \times(\mathbf{6} ; \mathbf{1})_{U} \\
2 \times(\mathbf{1} ; \mathbf{6})_{U} \\
4 \times(\mathbf{4} ; \mathbf{4})_{U} \\
10 \times(\mathbf{6} ; \mathbf{1})_{T} \\
4 \times(\mathbf{1 6} ; \mathbf{1})_{T} \\
2 \times(\mathbf{1} ; \mathbf{6})_{T} \\
2 \times(\mathbf{4} ; \mathbf{4})_{T}\end{array}$ & 20 & 0 \\
\hline
\end{tabular}

TABLE II. The massless spectra of the non-perturbative Type IIB orientifolds on $T^{4} / \mathbf{Z}_{6}$ with the NS-NS $B$ field of rank $b$. The semi-colon in the column "Charged Hypermultiplets" separates 99 and 55 representations. The subscript " $U$ " indicates that the corresponding ("untwisted") state is perturbative from the orientifold viewpoint. The subscript " $T$ " indicates that the corresponding ("twisted") state is non-perturbative from the orientifold viewpoint. The $U(1)$ charges are not shown, and by "neutral" hypermultiplets we mean that the corresponding states are not charged under the non-Abelian subgroups. Note that $\mathbf{1 6}$ is a reducible representation of $S U(4)$. 


\begin{tabular}{|c|c|c|c|c|c|}
\hline Model & $b \mid$ & Gauge Group & $\begin{array}{c}\text { Charged } \\
\text { Hypermultiplets }\end{array}$ & \begin{tabular}{|c|} 
Neutral \\
Hypermultiplets \\
\end{tabular} & \begin{tabular}{|c|} 
Extra Tensor \\
Multiplets
\end{tabular} \\
\hline $\mathbf{Z}_{4}, \mathrm{VS}$ & 2 & $\begin{array}{c}{[U(4) \otimes S p(4) \otimes S p(4)]_{99} \otimes} \\
{[U(4) \otimes S p(4) \otimes S p(4)]_{55}}\end{array}$ & \begin{tabular}{|l}
$(\mathbf{4}, \mathbf{4}, \mathbf{1} ; \mathbf{1}, \mathbf{1}, \mathbf{1})_{U}$ \\
$(\mathbf{4}, \mathbf{1}, \mathbf{4} ; \mathbf{1}, \mathbf{1}, \mathbf{1})_{U}$ \\
$(\mathbf{4}, \mathbf{1}, \mathbf{1} ; \mathbf{1}, \mathbf{4}, \mathbf{1})_{U}$ \\
$(\mathbf{4}, \mathbf{1}, \mathbf{1} ; \mathbf{1}, \mathbf{1}, \mathbf{4})_{U}$ \\
$2 \times(\mathbf{6}, \mathbf{1}, \mathbf{1} ; \mathbf{1}, \mathbf{1}, \mathbf{1})_{T}$ \\
$(\mathbf{1}, \mathbf{6}, \mathbf{1} ; \mathbf{1}, \mathbf{1}, \mathbf{1})_{T}$ \\
$(\mathbf{1}, \mathbf{1}, \mathbf{6} ; \mathbf{1}, \mathbf{1}, \mathbf{1})_{T}$ \\
$(\mathbf{4}, \mathbf{4}, \mathbf{1} ; \mathbf{1}, \mathbf{1}, \mathbf{1})_{T}$ \\
$(\mathbf{4}, \mathbf{1}, \mathbf{4} ; \mathbf{1}, \mathbf{1}, \mathbf{1})_{T}$ \\
same as above with $99 \leftrightarrow 55$
\end{tabular} & 18 & 2 \\
\hline $\mathbf{Z}_{4}, \mathrm{VS}$ & 4 & $\begin{array}{c}{[U(2) \otimes S p(2) \otimes S p(2)]_{99} \otimes} \\
{[U(2) \otimes S p(2) \otimes S p(2)]_{55}}\end{array}$ & \begin{tabular}{|l}
$(\mathbf{2}, \mathbf{2}, \mathbf{1} ; \mathbf{1}, \mathbf{1}, \mathbf{1})_{U}$ \\
$(\mathbf{2}, \mathbf{1}, \mathbf{2} ; \mathbf{1}, \mathbf{1}, \mathbf{1})_{U}$ \\
$2 \times(\mathbf{2}, \mathbf{1}, \mathbf{1} ; \mathbf{1}, \mathbf{2}, \mathbf{1})_{U}$ \\
$2 \times(\mathbf{2}, \mathbf{1}, \mathbf{1} ; \mathbf{1}, \mathbf{1}, \mathbf{2})_{U}$ \\
$2 \times(\mathbf{3}, \mathbf{1}, \mathbf{1} ; \mathbf{1}, \mathbf{1}, \mathbf{1})_{T}$ \\
$(\mathbf{1}, \mathbf{3}, \mathbf{1} ; \mathbf{1}, \mathbf{1}, \mathbf{1})_{T}$ \\
$(\mathbf{1}, \mathbf{1}, \mathbf{3} ; \mathbf{1}, \mathbf{1}, \mathbf{1})_{T}$ \\
$4 \times(\mathbf{2}, \mathbf{2}, \mathbf{1} ; \mathbf{1}, \mathbf{1}, \mathbf{1})_{T}$ \\
$4 \times(\mathbf{2}, \mathbf{1}, \mathbf{2} ; \mathbf{1}, \mathbf{1}, \mathbf{1})_{T}$ \\
same as above with $99 \leftrightarrow 55$ \\
$24 \times(\mathbf{1}, \mathbf{1}, \mathbf{1} ; \mathbf{1}, \mathbf{1}, \mathbf{1})_{T}$ \\
\end{tabular} & 17 & 3 \\
\hline $\mathbf{Z}_{6}, \mathrm{VS}$ & 4 & $\begin{array}{l}{[S p(4) \otimes S p(4)]_{99} \otimes} \\
{[S p(4) \otimes S p(4)]_{55}}\end{array}$ & \begin{tabular}{|l}
$(\mathbf{4}, \mathbf{4} ; \mathbf{1}, \mathbf{1})_{U}$ \\
$(\mathbf{1}, \mathbf{1} ; \mathbf{4}, \mathbf{4})_{U}$ \\
$2 \times(\mathbf{4}, \mathbf{1} ; \mathbf{4}, \mathbf{1})_{U}$ \\
$2 \times(\mathbf{1}, \mathbf{4} ; \mathbf{1}, \mathbf{4})_{U}$ \\
$5 \times(\mathbf{6}, \mathbf{1} ; \mathbf{1}, \mathbf{1})_{T}$ \\
$5 \times(\mathbf{1}, \mathbf{6} ; \mathbf{1}, \mathbf{1})_{T}$ \\
$4 \times(\mathbf{4}, \mathbf{4} ; \mathbf{1}, \mathbf{1})_{T}$ \\
$(\mathbf{1}, \mathbf{1} ; \mathbf{6}, \mathbf{1})_{T}$ \\
$(\mathbf{1}, \mathbf{1} ; \mathbf{1}, \mathbf{6})_{T}$ \\
$(\mathbf{4}, \mathbf{1} ; \mathbf{4}, \mathbf{1})_{T}$ \\
$(\mathbf{1}, \mathbf{4} ; \mathbf{1}, \mathbf{4})_{T}$
\end{tabular} & 20 & 0 \\
\hline
\end{tabular}

TABLE III. The massless spectra of the non-perturbative Type IIB orientifolds on $T^{4} / \mathbf{Z}_{N}$ for $N=4,6$ with the NS-NS $B$ field of rank $b$. The semi-colon in the column "Charged Hypermultiplets" separates 99 and 55 representations. The subscript " $U$ " indicates that the corresponding ("untwisted") state is perturbative from the orientifold viewpoint. The subscript " $T$ " indicates that the corresponding ("twisted") state is non-perturbative from the orientifold viewpoint. The $U(1)$ charges are not shown, and by "neutral" hypermultiplets we mean that the corresponding states are not charged under the non-Abelian subgroups. Note that $\mathbf{6}$ is a reducible representation of $S p(4)$ (in our conventions the rank of $S p(2 n)$ is $n$ ). 


\section{FIGURES}

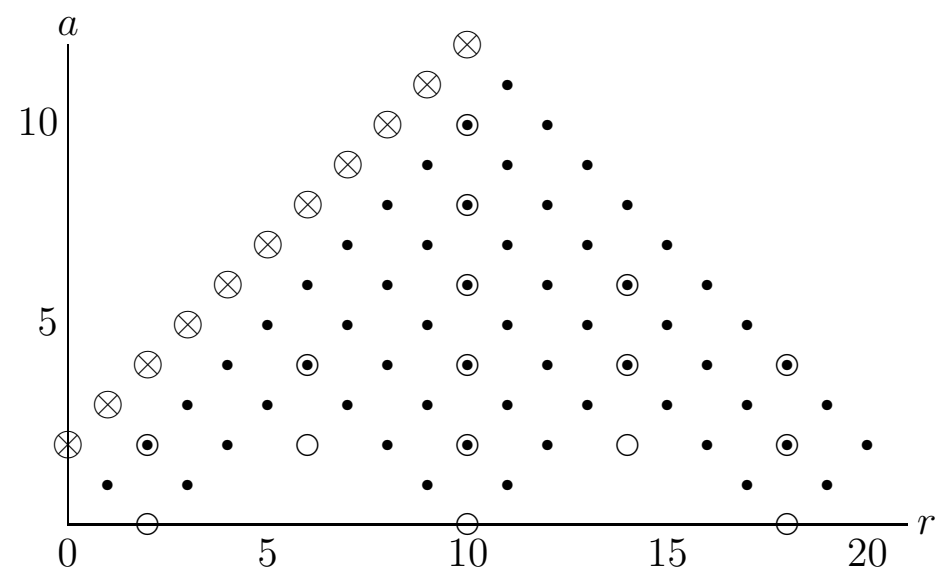

FIG. 1. Open circles and dots represent the original Voisin-Borcea orbifolds. The line of $\otimes$ 's corresponds to the extension discussed in the text. 


\section{REFERENCES}

[1] G. Pradisi and A. Sagnotti, Phys. Lett. B216 (1989) 59;

M. Bianchi and A. Sagnotti, Phys. Lett. B247 (1990) 517; Nucl. Phys. B361 (1991) 539.

[2] E.G. Gimon and J. Polchinski, Phys. Rev. D54 (1996) 1667.

[3] E.G. Gimon and C.V. Johnson, Nucl. Phys. B477 (1996) 715.

A. Dabholkar and J. Park, Nucl. Phys. B477 (1996) 701.

[4] Z. Kakushadze, G. Shiu and S.-H.H. Tye, Phys. Rev. D58 (1998) 086001.

[5] M. Berkooz and R.G. Leigh, Nucl. Phys. B483 (1997) 187.

[6] C. Angelantonj, M. Bianchi, G. Pradisi, A. Sagnotti and Ya.S. Stanev, Phys. Lett. B385 (1996) 96.

[7] Z. Kakushadze, Nucl. Phys. B512 (1998) 221.

[8] Z. Kakushadze and G. Shiu, Phys. Rev. D56 (1997) 3686; Nucl. Phys. B520 (1998) 75.

[9] G. Zwart, Nucl. Phys. B526 (1998) 378.

[10] G. Aldazabal, A. Font, L.E. Ibáñez and G. Violero, Nucl. Phys. B536 (1998) 29.

[11] Z. Kakushadze, G. Shiu and S.-H.H. Tye, Nucl. Phys. B533 (1998) 25.

[12] Z. Kakushadze, Phys. Lett. B434 (1998) 269; Nucl. Phys. B535 (1998) 311; Phys. Rev. D58 (1998) 101901.

[13] Z. Kakushadze, hep-th/9904007.

[14] Z. Kakushadze, hep-th/9904211.

[15] J. Polchinski, Phys. Rev. D55 (1997) 6423.

[16] J.D. Blum, Nucl. Phys. B486 (1997) 34.

[17] M. Bianchi, G. Pradisi and A. Sagnotti, Nucl. Phys. B376 (1992) 365.

[18] Z. Kakushadze, Nucl. Phys. B544 (1999) 265.

[19] E. Witten, JHEP 9802 (1998) 006.

[20] A. Sen and S. Sethi, Nucl. Phys. B499 (1997) 45.

[21] M. Berkooz, R.G. Leigh, J. Polchinski, J.H. Schwarz, N. Seiberg and E. Witten, Nucl. Phys. B475 (1996) 115.

[22] K. Intriligator, Nucl. Phys. B496 (1997) 177;

J.D. Blum and K. Intiligator, Nucl. Phys. B506 (1997) 223.

[23] M.B. Green, J.H. Schwarz and P.C. West, Nucl. Phys. B254 (1985) 327;

J. Erler, J. Math. Phys. 35 (1994) 1819.

[24] A. Sagnotti, Phys. Lett. B294 (1992) 196.

[25] S. Kachru and C. Vafa, Nucl. Phys. B450 (1995) 69.

[26] C. Vafa, Nucl. Phys. B469 (1996) 285.

[27] C. Voisin, in: Jorneés de Géometrie Algébrique d'Orsey, eds. A. Beauville et al., Astérisque, Vol. 218 (Soc. Math. France, 1993) p.273.

[28] C. Borcea, in: Mirror Manifolds II, eds. B.R. Greene and S.-T. Yau (International Press and AMS, 1997) p.717.

[29] D.R. Morrison and C. Vafa, Nucl. Phys. B473 (1996) 74; Nucl. Phys. B476 (1996) 437.

[30] P.S. Aspinwall, Nucl. Phys. 460 (1996) 57.

[31] V.V. Nikulin, in: Proc. ICM (Berkeley, 1986) p.654.

[32] A. Font, L.E. Ibáñez and F. Quevedo, Phys. Lett. B217 (1989) 272.

[33] C. Vafa and E. Witten, J. Geom. Phys. 15 (1995) 189.

[34] E. Perevalov and G. Rajesh, Phys. Rev. Lett. 79 (1997) 2931. 
[35] E.G. Gimon and C.V. Johnson, Nucl. Phys. B479 (1996) 285.

[36] P. Berglund and E.G. Gimon, Nucl. Phys. B525 (1998) 73. 